\title{
Dopamine beta-hydroxylase deficiency
}

INSERM

\section{Source}

INSERM. (1999). Orphanet: an online rare disease and orphan drug data base. Dopamine beta-hydroxylase deficiency. ORPHA:230

Dopamine beta-hydroxylase deficiency is an extremely rare genetic metabolic disorder characterized by autonomic dysregulation leading mainly to orthostatic hypotension. 